\title{
Associazione Americana di Endocrinologia Clinica e Associazione Medici Endocrinologi: algoritmo per la diagnostica dei noduli tiroidei
}

\author{
Maria Grazia Castagna ${ }^{1}$
}

Accettato: 23 settembre 2021 / Pubblicato online: 28 gennaio 2022

(c) The Author(s) 2022

Commento a:

American Association of Clinical Endocrinology and Associazione Medici Endocrinologi thyroid nodule algorithmic tool.

J.R. Garber, E. Papini, A. Frasoldati, M.A. Lupo, R.M. Harrell, S. Parangi, V. Patkar, Z.W. Baloch, R. Pessah-Pollack, L. Hegedus, A. Crescenzi, C.C. Lubitz, R. Paschke, G.W. Randolph, R. Guglielmi, C.P. Lombardi, H. Gharib.

Endocr Pract (2021) 27(7):649-660

La storia naturale dei noduli tiroidei e dei tumori della tiroide evidenzia come la maggior parte dei noduli tiroidei siano benigni e come molti tumori della tiroide siano indolenti, con un tasso di mortalità molto basso. Le recenti linee guida sul nodulo hanno cercato di ottimizzarne la gestione, adottando un approccio diagnostico più aggressivo per noduli tiroidei ad alto rischio di cancro e riservando il solo monitoraggio ecografico ai noduli definiti a basso rischio, sulla base delle loro caratteristiche ecografiche $[1,2]$.

Il lavoro di Garber e collaboratori [3] descrive lo sviluppo e le caratteristiche di uno strumento interattivo che può aiutare il clinico nella valutazione iniziale e nel trattamento dei pazienti con noduli tiroidei.

Un gruppo di lavoro, che ha coinvolto l'American Association of Clinical Endocrinology (AACE) e l'Associazione Medici Endocrinologi (AME), si è incontrato periodicamente per circa 3 anni e ha sviluppato un algoritmo per la gestione del nodulo tiroideo.

L'algoritmo tiene conto di informazioni cliniche (storia familiare di cancro midollare della tiroide o sindrome ereditaria che predispone al cancro della tiroide, noduli tiroidei captanti al fluoridesossiglucosio, aumento della calcitonina

M.G. Castagna

mariagrazia.castagna@unisi.it

1 Dipartimento di Scienze Mediche, Chirurgiche e Neuroscienze, Università di Siena, Siena, Italia sierica, nodulo duro e/o fisso all'esame obiettivo, adenopatia sospetta), caratteristiche ecografiche e citologiche del nodulo. È stato assegnato un punteggio numerico ad ogni caratteristica del nodulo sulla base del rischio associato di malignità. Un algoritmo elettronico chiamato TNAPP (App per noduli tiroidei) è stato il prodotto finale ed è disponibile online all'indirizzo https://aace-thyroid.deontics.com.

I dati da inserire includono:

- fattori clinici (26 variabili) che pesano per o contro l'esecuzione dell'agoaspirato tiroideo. Questi possono essere inseriti, ma non sono obbligatori;

- caratteristiche ecografiche (36 variabili in 9 domande) quali le dimensione del nodulo, la sua composizione ed ecogenicità. Ulteriori dati possono essere inseriti quali la forma del nodulo (ad esempio, più alto che largo), margini, vascolarizzazione, aspetto della componente solida e calcificazioni;

- caratteristiche citologiche (48 variabili in 4 domande) sono facoltativamente inseriti se è stata eseguita la valutazione citologica e include le categorie Bethesda (I-VI).

Dai dati di input forniti, il TNAPP fornisce i seguenti output all'utente:

- la categoria di rischio ecografico del nodulo basata sulle indicazioni riportate nelle linee guida dell'American Thyroid Association. Se vengono inseriti dettagli sufficienti, viene anche fornita la classe di rischio ecografica secondo l'American College of Radiology e secondo l'ACR-TIRADS;

- l'indicazione a effettuare la valutazione citologica e/o quando ripetere il monitoraggio ecografico;

- la gestione del nodulo sulla base dell'esito della citologia, se effettuata;

- la probabilità (espressa in percentuale) di malignità all'istologia, sulla base del risultato citologico.

Non c'è dubbio che la valutazione del nodulo tiroideo sia diventata sempre più complessa e richieda l'integrazione di dati clinici, biochimici, ecografici, citologici e molecolari. Questa attenta valutazione è finalizzata alla stratificazione del rischio di malignità, al fine di evitare un trattamento chi- 
rurgico non necessario in caso di patologie benigne o indolenti $[1,2,4]$. TNAPP, come descritto da Garber e colleghi [3], fornisce uno strumento di supporto in grado di integrare tutte le informazioni disponibili e fornire al clinico il rischio di malignità e le modalità di gestione del nodulo. Lo sviluppo di TNAPP segna una vera innovazione e questa app può essere facilmente diffusa, utilizzata e modificata in futuro sulla base di nuove evidenze scientifiche.

Tuttavia, lo studio ha validato l'APP solo su una casistica di 95 noduli. Si rende necessaria, quindi, una validazione retrospettiva e prospettica su una coorte di noduli tiroidei più ampia per confermarne l'utilità e sicurezza nella pratica clinica.

Open Access This article is licensed under a Creative Commons Attribution 4.0 International License, which permits use, sharing, adaptation, distribution and reproduction in any medium or format, as long as you give appropriate credit to the original author(s) and the source, provide a link to the Creative Commons licence, and indicate if changes were made. The images or other third party material in this article are included in the article's Creative Commons licence, unless indicated otherwise in a credit line to the material. If material is not included in the article's Creative Commons licence and your intended use is not permitted by statutory regulation or exceeds the permitted use, you will need to obtain permission directly from the copyright holder. To view a copy of this licence, visit http://creativecommons.org/licenses/by/4.0/.

\section{Bibliografia}

1. Haugen BR, Alexander EK, Bible KC et al (2016) 2015 American Thyroid Association management guidelines for adult patients with thyroid nodules and differentiated thyroid cancer: the American Thyroid Association guidelines task force on thyroid nodules and differentiated thyroid cancer. Thyroid 26:1-131

2. Gharib H, Papini E, Garber JR et al(AACE/ACE/AME Task Force on Thyroid Nodules) (2016) American Association of Clinical Endocrinologists, American College of Endocrinology, and Associazione Medici Endocrinologi medical guidelines for clinical practice for the diagnosis and management of thyroid nodules - 2016 update. Endocr Pract 22:622-639

3. Garber JR, Papini E, Frasoldati A et al (2021) American Association of Clinical Endocrinology and Associazione Medici Endocrinologi thyroid nodule algorithmic tool. Endocr Pract 27:649-660

4. Tessler FN, Middleton WD, Grant EG et al (2017) ACR Thyroid Imaging, Reporting and Data System (TI-RADS): white paper of the ACR TI-RADS Committee. J Am Coll Radiol 14:587-595

Nota della casa editrice Springer Nature rimane neutrale in riguardo alle rivendicazioni giurisdizionali nelle mappe pubblicate e nelle affiliazioni istituzionali. 